\title{
Estimating the position and orientation of a mobile robot using neural network framework based on combined square-root cubature Kalman filter and simultaneous localization and mapping
}

\author{
Wang, D. ${ }^{a}$, , Tan, K. ${ }^{a}$, Dong, Y. ${ }^{a}$, Yuan, G. ${ }^{b}$, Du, X. ${ }^{b}$ \\ ${ }^{a}$ College of Electronic Information and Electrical Engineering, Anyang Institute Of Technology, Anyang Henan, P.R. China \\ ${ }^{b}$ College of Automation, Harbin Engineering University, Harbin Heilongjiang, P.R. China
}

\begin{abstract}
A B S T R A C T
The real-time performance of target tracking, detection, and positioning behaves not well for non-Gaussian and nonlinear model with circumstance uncertainty. The weak observability of the system under large noise causes the algorithm unstable and slow to converge. A new estimation algorithm combining square-root cubature Kalman filter (SRCKF) with simultaneous localization and mapping (SLAM) is proposed. By connecting neural network weights, network input, functional types and ideal output network, the algorithm firstly update iteratively the SRCKF-SLAM state model and observation model, then conduct the cubature point estimate (weights) neural network framework. Thus, a point set better representing the target state and a more accurate state estimation are achieved, which can improve the filtering accuracy. This paper also estimates robot and characteristic states by filtering in groups. The simulation results showed that the proposed algorithm is feasible and effective. Compared with other filtering algorithms such as SRUKF and SRCDKF, it improves the estimation accuracy. Applying the new algorithm to the position filtering estimation of mobile robot can effectively reduce the positioning error, achieve high-precision tracking detection, and improve the accuracy of robot target detection.
\end{abstract}

\author{
ARTICLE INFO \\ Keywords: \\ Robot; \\ Mobile robot; \\ Square-root cubature Kalman \\ filter; Simultaneous localization \\ and mapping; \\ Sensors; \\ Artificial neural networks; \\ Iteration update; \\ Filter estimate \\ *Corresponding author: \\ lansejingling1988@126.com \\ (Wang, D.) \\ Article history: \\ Received 9 January 2020 \\ Revised 15 March 2020 \\ Accepted 17 March 2020
}

\section{References}

[1] Smith, R., Self, M., Cheeseman, P. (1988). Estimating uncertain spatial relationships in robotics, In: Lemmer, J.F., Kanal, L.N. (eds.), Machine Intelligence and Pattern Recognition, Amsterdam North-Holland, Vol. 5, 435-461, doi: 10.1016/B978-0-444-70396-5.50042-X.

[2] Yuan, G.-N., Wang, D.-D., Wei, Y.-H., Hong, W. (2013). Particle filter SLAM algorithm for underwater oil pipeline leakage detection and positioning, Journal of Chinese Inertial Technology, Vol. 21, No. 2, 204-208.

[3] Sun, Y., Lu, D., Chen, Q. (2013). An improved cubature Kalman filters based on strong tracking, Journal of Huazhong University of Science and Technology (Natural Science Edition), Vol. 41, 451-454.

[4] Wang, H., Fu, G., Li, J., Yan, Z., Bian, X. (2013). An adaptive UKF based SLAM method for unmanned underwater vehicle, Mathematical Problems in Engineering, Vol. 2013, Article ID 605981, doi: 10.1155/2013/605981.

[5] Yuan, G., Wang, D., Tan, K. (2013). SLAM algorithm based on the grid map fuzzy logic, Journal of Huazhong University of Science and Technology (Natural Science Edition), Vol. 41, No. 9, 32-36.

[6] Yuan, G., Hu, Z., Zhang, J., Zhao, X., Fu, C. (2016) Novel neural network framework based on iterated cubature Kalman filter, Computer Science, Vol. 43, No. 10, 256-261. 
[7] Feng, K., Li, J., Zhang, X., Zhang, X., Shen, C., Cao, H., Yang, Y., Liu, J. (2018). An improved strong tracking cubature Kalman filter for GPS/INS integrated navigation systems, Sensors, Vol. 18, No. 6, 1919-1940, doi: $10.3390 / \mathrm{s} 18061919$.

[8] Yue, C., Bo, Y.-M., Wu P.-L., Tian, M.-C., Chen, Z.-M. (2017). Non-cooperative space target tracking based on fuzzy iterative square-root cubature Kalman filter, Journal of Chinese Inertial Technology, Vol. 25, No. 3, 395-398.

[9] Gao, W., Zhang, Y., Sun, Q., Guan, J. (2014). Simultaneous localization and mapping based in iterated square root cubature Kalman filter, Journal of Harbin Institute of Technology, Vol. 46, No. 12, 120-124.

[10] Adam, S.P., Karras, D.A., Magoulas, G.D., Vrahatis, M.N. (2014). Solving the linear interval tolerance problem for weight initialization of neural networks, Neural Networks, Vol. 54, 17-37, doi: 10.1016/j.neunet.2014.02.006.

[11] Kim, K.J., Park, J.B., Choi, Y.H. (2006). The adaptive learning rates of extended Kalman filter based training algorithm for wavelet neural networks, In: Gelbukh A., Reyes-Garcia C.A. (eds.), MICAI 2006: Advances in Artificial Intelligence, MICAI 2006, Lecture Notes in Computer Science, Vol. 4293, 327-337, Springer, Berlin, Germany, doi: $10.1007 / 1192523131$.

[12] Li, W., Fang, J. (2014). BP neural network for mobile robot self-tuning PID controller PID controller design, $A d$ vanced Materials Research, Vol. 898, 755-758, doi: 10.4028/www.scientific.net/AMR.898.755.

[13] Ahmad, M.A., Azuma, S.-I., Sugie, T. (2014). Performance analysis of model-free PID tuning of MIMO systems based on simultaneous perturbation stochastic approximation, Expert Systems with Applications, Vol. 41, No. 14, 6361-6370, doi: 10.1016/j.eswa.2014.03.055.

[14] Du, X., Zhao, Y., Yuan, G., Xia, G., Chang, S. (2014). A novel initial method of fuzzy wavelet network in oncethrough steam generator control, In: Proceedings of the 33rd Chinese Control Conference, Nanjing, China, 86938698, doi: 10.1109/ChiCC.2014.6896461.

[15] Sadati, N., Ghaffarkhah, A., Ostadabbas, S. (2008). A new neural network based FOPID controller, In: Proceedings of 2008 IEEE International Conference on Networking, Sensing and Control, Sanya, China, 762-767, doi: 10.1109/ ICNSC.2008.4525318.

[16] Chatterjee, S., Sadhu, S., Ghoshal, T.K. (2017). Improved estimation and fault detection scheme for a class of nonlinear hybrid systems using time delayed adaptive CD state estimator, IET Signal Processing, Vol. 11, No. 7, 771779, doi: 10.1049/iet-spr.2016.0380.

[17] Al-Shabi, M. (2017). Sigma-point smooth variable structure filters applications into robotic arm, In: 7th International Conference on Modeling, Simulation, and Applied Optimization (ICMSAO), Sharjah, United Arab Emirates, doi: 10.1109/ICMSA0.2017.7934865.

[18] Li, J., Zhang, J.-X., Zhang, Y.-H., Chen, L.-J. (2017). Estimation of vehicle state and parameter based on strong tracking CDKF, Journal of Jilin University Engineering and Technology Edition, Vol, 47, No. 5, 1329-1335, doi: 10.13229/i.cnki.jdxbgxb201705001.

[19] Wang, G., Li, N., Zhang, Y. (2016). Diffusion distributed Kalman filter over sensor networks without exchanging raw measurements, Signal Processing, Vol. 132, 1-7, doi: 10.1016/i.sigpro.2016.07.033.

[20] Chen, M. (2017). The sequential WSNs target tracking algorithm based on adaptive SR-CKF, Chinese Journal of Sensors and Actuators, Vol. 30, No. 8, 1220-1225, doi: 10.3969/j.issn.1004-1699.2017.08.016. 


\section{APEM}

\title{
Ocenjevanje položaja in usmerjenosti mobilnega robota z uporabo nevronske mreže na podlagi kombinacije kvadratno-korenskega kubaturnega Kalmanovega filtra in sočasne lokalizacije in preslikave
}

\author{
Wang, D. ${ }^{a}{ }^{,}$, Tan, K. ${ }^{a}$, Dong, Y. ${ }^{a}$, Yuan, G. ${ }^{b}$, Du, X. ${ }^{b}$ \\ ${ }^{a}$ College of Electronic Information and Electrical Engineering, Anyang Institute Of Technology, Anyang Henan, P.R. China \\ ${ }^{b}$ College of Automation, Harbin Engineering University, Harbin Heilongjiang, P.R. China
}

\begin{abstract}
POVZETEK
Zmogljivost sledenja, odkrivanja in določanja položaja v realnem času je nezanesljiva $\mathrm{v}$ ne-Gaussovem in nelinearnem modelu $\mathrm{z}$ negotovostjo okoliščin. Šibka opaznost sistema pri velikem šumu povzroči, da algoritem nestabilno in počasi konvergira. Predlagan je nov algoritem ocenjevanja, ki združuje kvadratno-korenski kubaturni Kalmanov filter (SRCKF) s sočasno lokalizacijo in preslikavo (SLAM). S povezovanjem uteži nevronske mreže, vhodnih podatkov, funkcionalnih tipov in idealne izhodne mreže, algoritem najprej iterativno posodobi model stanja in model opazovanja SRCKF-SLAM in nato vzpostavi okvir nevronske mreže za oceno kubature (uteži). Tako se najde nabor točk, ki bolje predstavljajo ciljno stanje, in podajajo natančnejšo oceno stanja, kar lahko izboljša natančnost filtriranja. Ta članek, s filtriranjem po skupinah ocenjuje tudi robota in značilna stanja. Rezultati simulacije so pokazali, da je predlagani algoritem izvedljiv in učinkovit. V primerjavi z drugimi algoritmi filtriranja, kot sta SRUKF in SRCDKF, izboljša natančnost ocenjevanja. Uporaba novega algoritma pri oceni filtriranja položaja mobilnega robota lahko učinkovito zmanjša napako pozicioniranja, doseže visoko natančnost sledenja in izboljša natančnost zaznavanja ciljev robota.
\end{abstract}

(C) 2020 CPE, University of Maribor. All rights reserved.

\section{PODATKI O ČLANKU}

Ključne besede:

Robot;

Mobilni robot;

Kvadratno-korenski kubaturni

Kalmanov filter;

Sočasna lokalizacija in preslikava;

Senzorji;

Umetne nevronske mreže;

Posodobitev iteracije;

Ocena filtra

*Kontaktna oseba:

lansejingling1988@126.com

(Wang, D.)

Zgodovina članka:

Prejet 9. januarja 2020

Popravljen 15. marca 2020

Sprejet 17. marca 2020 